\title{
Cysticercal Encephalitis, A Rare Presentation of Neurocysticercosis: A Case Report
}

\author{
BITHI DEBNATH ${ }^{1}$, SURAJ C MAZUMDER ${ }^{2}$, MUSTAFA MAHBUB $^{3}$, NAILAZAMAN KHAN ${ }^{4}$
}

\begin{abstract}
Introduction
Neurocysticercosis is the most common parasitic infection of the central nervous system caused by the larval form of the Tape worm Taenia Solium. It is widely prevalent in developing countries of Africa, Asia and Latin America. ${ }^{1}$ In India, cysticercosis is highly prevalent and CNS involvement is seen in $60-90 \%$ of infested patients. ${ }^{2}$

The clinical manifestations of the disease may vary with the size and location of cysticerci and the intensity of the host's immune response. Acute symptomatic seizures are the most common manifestations. The other clinical conditions include headache, hydrocephalus, chronic meningitis, focal neurological deficits, psychological disorders and raised intracranial pressure. ${ }^{3}$ Altered sensorium and raised intracranial pressure (ICP) may require ventilatory support in an intensive care unit (ICU).
\end{abstract}

\section{Case Summary}

A 4 years 6 months old boy was brought to emergency department with complaints of low grade fever, headache, altered sensorium and generelized tonic-clonic seizure for 3 days. He was born and lived in Hatiya, Noakhali. Parents reported no weight loss or other symptoms of concern before this illness. He had not been exposed to pork and family history was silent for epilepsy. There was no history of recent immunization, ear discharge or tuberculosis contact. The child on admission was febrile, had Glasgow coma scale 8 without any features of meningeal irritation. His pupils were normal in size and reacting normally to light. On motor examination, all four limbs had power of grade III/V,

1. FCPS student (child neurology \& development), Department of Neurosciences, Dhaka Shishu Hospital, Dhaka.

2. Registrar, Department of Neurosciences, Dhaka Shishu Hospital, Dhaka.

3. Associate Professor, Department of Neurosciences, Bangladesh institute of child health (BICH), Dhaka Shishu Hospital, Dhaka.

4. Professor, Department of Neurosciences, Bangladesh institute of child health (BICH), Dhaka Shishu Hospital, Dhaka Correspondence: Dr. Bithi Debnath, Email: bithidebnath @gmail.com exaggerated deep tendon reflexes and bilateral extensor plantar response. Rest of the systemic examination was essentially normal. Fundus examination revealed bilateral papilledema.

Then the child was transferred to ICU due to low GCS and phenobarbiton loading dose $(20 \mathrm{mg} / \mathrm{kg}$ ) followed by maintenance $(5 \mathrm{mg} / \mathrm{kg} / \mathrm{day})$ was administered along with mannitol. The patient was also managed with inj. Dexamethasone, broad spectrum antibiotics and antivirals inj. Acyclovir. Laboratory tests showed leukocytosis with mild eosinophilia. His x-ray's chest, blood glucose, electrolytes, liver, renal function tests and blood ammonia levels were normal. Mantoux test was negative.

EEG recorded severe generelized encephalopathy. His sensorium was gradually improved and we did Cerebrospinal fluid study which showed 16 cells $/ \mathrm{mm} 3$ (100\% lymphocytes) with protein, $40 \mathrm{mg} / \mathrm{dl}$ and sugar $58 \mathrm{mg} / \mathrm{dl}$. CSF culture was sterile . MRI of brain which was done on day $8^{\text {th }}$ of admission revealed mutiple hypointense (T1W) (Fig-1A) and hyperintense (T2W, FLAIR) (Fig-1B, Fig-1C) lesions in both centrum semi ovale, para ventricular region, both fronto parietal, occipital lobe and thalamus. After contrast, rim enhancement(Fig-1D) of the lesions were noted. Features were suggestive for neurocysticercosis.

After getting CSF and MRI findings, the child was started oral prednisolone ( $2 \mathrm{mg} / \mathrm{kg} /$ day) and was continued for 2 weeks. Albendazole therapy $(15 \mathrm{mg} /$ $\mathrm{kg} /$ day) was started later with prednisolone and continued for 4 weeks. Regular physiotherapy was also given. He was seizure free since $4^{\text {th }}$ day of admission. The boy was hospitalized for about 1 month and on discharge, he was alert, oriented, seizure free but persisting quadriparesis. A month after discontinuation of definitive treatment, EEG recordings were normal and head CT scan demonstrated complete resolution of parenchymal cyst (Fig-2). Gradually antiepileptic therapy was discontinued and during the following 6 months the patient remained seizure free and to date, the boy presents good general conditions. 


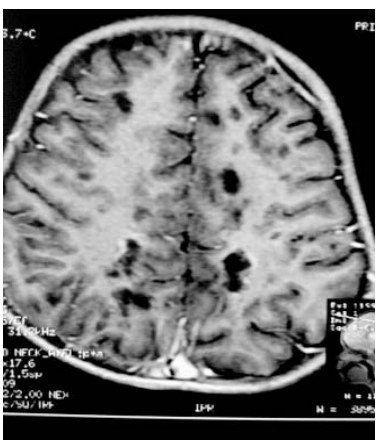

A

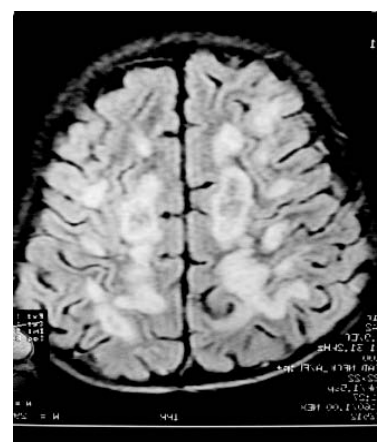

C

Fig-1: $M R I$ of brain of the patient. $T_{1}$ iso to hypo intense (A), in $T_{2}(B)$ and FLAIR (C) multiple hyper intense lesions are noted in both centrum semi ovale, paraventricular region, both fronto parietal lobe and thalamus. After contrast rim enhancement (D) of few of the lesions are noted.

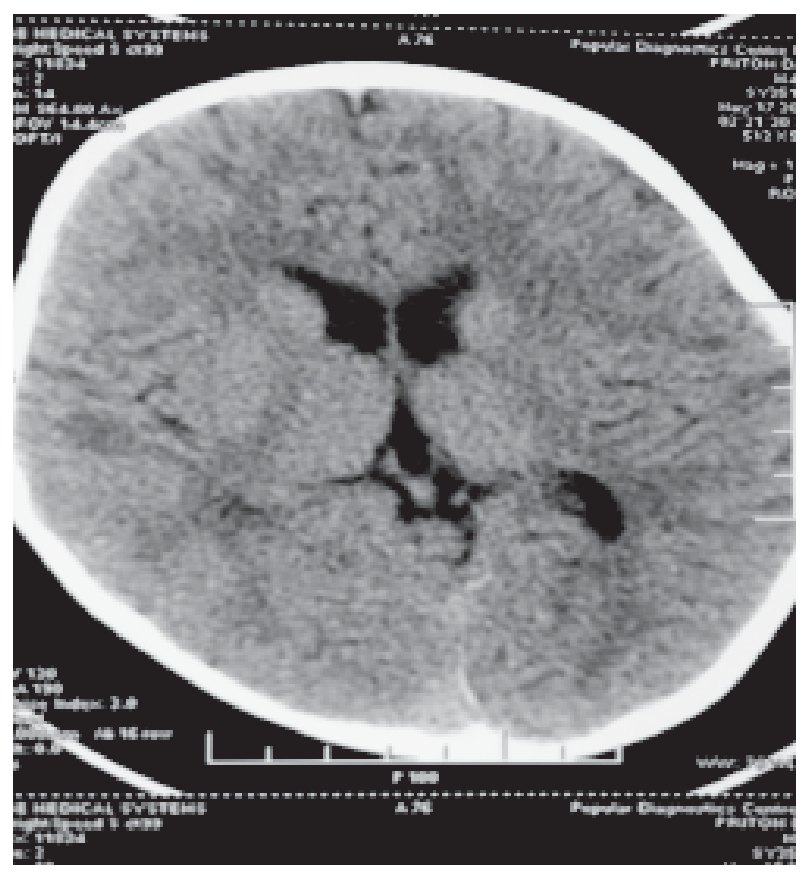

Fig-2: CT scan of head of the patient showed complete resolution of parenchymal cyst.
Table-I

Revised Diagnostic Criteria for Neuro-cysticercosis.

\section{Absolute}

Histological demonstration of the parasite from biopsy of brain or spinal cord lesion

Cystic lesions with scolex on CT or MRI

Direct visualization of subretinal parasite by fundoscopy

Major

Lesions highly suggestive of NCC on neuroimaging*

Positive serum EITB for detection of anti-cysticercal antibodies

Resolution of cysts after antiparasitic therapy

Spontaneous resolution of small single enhancing lesions

\section{Minor}

Lesions compatible with NCC on neuroimaging\#

Clinical manifestations suggestive of NCC**

Positive CSF-ELISA for detection of anticysticereal antibodies or cysticercal antigens

Cysticercosis outside the CNS

\section{Epidemilologic}

Evidence of household contact with Tanca solium infection.

Individual coming from living in an endemic area.

History of navel to an endemic area.

Reproduced from Ref. 6 with permission.

${ }^{\star} \mathrm{CT}$ or MRI showing cystic lesions without scolex, enhancing lesions or typical brain calcifications.

\#CT/MRI showing hydrocephalus or abnormal enhancement of meninges and myelogram showing filling defect.

**seizores, focal signs, intracranial hypertension and dementia.

Definitive: Presence of one absolute criterion or two major plus one minor and one epidemiologic criteria.

Probable: Presence of one major plus two minor criteria or one major plus one minor and one epidemiologic criteria or three minor plus one epidemiologic criteria. 


\section{Discussion}

Neurocysticercosis (NCC) is the most common CNS parasitosis worldwide. It accounts for about 50000 death per year. In disease-endemic areas, about a third of all cases of epilepsy are due to neurocysticercosis. ${ }^{4}$

Cysticercosis is acquired by ingesting food or water contaminated with eggs of $T$. solium. Rarely infection may occur after autoingestion of eggs in patients who have intestinal tapeworms. As in pigs, in the human intestine the eggs hatch to release larvae that penetrate the intestinal mucosa and migrate throughout the body to produce human cysticercosis. Although cysts may be found in any human tissue, most mature cysts are found in CNS, skeletal muscle, subcutaneous tissue and eyes. In CNS, encysted larvae preferably locate in areas with high blood flow such as grey-white matter junction. ${ }^{5}$

Most patients with neurocysticercosis have few intracranial lesions. However, a small subset show development of massive infections that may be divided into encephalitic and nonencephalitic. Proper differentiation of both forms is important because they have different pathogenetic mechanisms and require different therapeutic approaches. The encephalitic form often occurs in children and young women who have not had contact with the parasite until they are infected with a heavy load of Taenia solium eggs. In these cases, the host's immune system actively reacts against the parasites. Intracranial hypertension occurs in patients with Cysticercotic encephalitis which is characterized by clouding of consciousness, seizures, diminution of visual acuity, headache, vomiting, and papilledema.$^{6}$ Other unusual manifestations of NCC are stroke, visual loss, ataxia, dystonia, dementia and hydrocephalus. ${ }^{7,8}$ Cysticercal granuloma as cause of Weber's syndrome and ptosis $^{9}$ have also been reported. Our patient presented with features of raised intracranial pressure. He fulfilled the diagnostic criteria of neurocysticercosis. ${ }^{10}$ Prasad et al reported a case of cysticercal encephalitis with cortical blindness. ${ }^{11}$

MRI is considered the best neuroimaging tool for the detection of degenerating and innocuous (viable) cysticerci, while CT is better for calcified lesions. ${ }^{3}$ In our patient, MRI of brain was done at $8^{\text {th }}$ day of admission. By that time patient had already got mannitol and dexamethasone. That's why brain oedema might be decreased. Analysis of CSF showed abnormalities which was suggestive of encephalitis. Enzyme-linked immunoelctrotransfer blot (EITB) with $100 \%$ sensitivity and an overall $98 \%$ specificity is commonly used to detect specific antibodies. ${ }^{3}$ But we had no fascilities to do that test at that time. Tissue biopsy showing cysticercus larvae remains the most accurate means of diagnosis. ${ }^{10}$

In patients with intracranial hypertension secondary to NCC, the priority is to manage the raised ICP before considering other treatment options. Antiparasitic drug treatment is never the primary treatment modality, especially in the setting of elevated ICP. ${ }^{12}$ Therefore, all patients with multiple cysts should receive treatment with steroid to reduce intracranial pressure first and then cysticidal drug, that is, albendazole. Efficacy of treatment should be monitored by repeat CT and MRI after 3 months. ${ }^{11}$ Treatment with albendazole, $15 \mathrm{mg} / \mathrm{kg} /$ day in 2-3 doses for $28 \mathrm{~d}$, is considered to be effective for cyst destruction. Nevertheless, shorter durations of 14 and $8 d$ have also been used and demonstrated to be equally effective. ${ }^{13-15}$

\section{Conclusion}

The present report reminds us to consider NCC as a possible cause of encephalitis specially in children living in developing countries like Bangladesh. Proper diagnosis and appropriate therapy can result in better outcome in this rare but commonly fatal neurological disorder. NCC is a preventable disease; however, control of raised intracranial pressure and seizures are the mainstay of management during acute presentations.

\section{References}

1. Coyle CM, Mahanty S, Zunt JR. Neurocysticercosis: neglected but not forgotten. PLoS Negl Trop Dis 2012;6:e1500

2. Hawk MW, Shahlaie K, Kim KD. Neurocysticercosis: a review. Surg Neurol 2005;63:123-32.

3. Garcia HH, Gonzalez AE, Evans CA, Gilman $\mathrm{RH}$. Cysticerosis Working Group in Peru. Taenia solium cysticercosis. Lancet 2003;362:547-56.]

4. RomanG, Sotelo J, Brutto O. A proposal to declare neurocysticercosis an international reportable disease. Bull World Health Organ 2000;78:399-406. 
5. Giacomet V, Penagini F, Erba P, Nannini P, Pisanelli S, Ramponi G. Neurocysticercrsis in a 14year old boy in Italy: An unexpected case. Asian Pac J Trop Dis 2013;3(4):324-26.

6. Rangel R, Torres B, Del Bruto O, Sotelo J. Cysticercotic encephalitis: a severe form in young females. Am J Trop Med Hyg 1987; 36: 387-92.

7. Singhi $P$, Ray M, Singhi S. Clinical spectrum of 500 children with neurocysticercosis and response to albendazole therapy. J Child Neurol 2000;15:207-13.

8. Jha S, Kumar R, Kumar R. Clinical pleomorphism of neurocysticercosis in children: some unusal manifestations. J Pediatr Neurol 2006;4:103-9.

9. Singhi P, Mahajan V, Khandelwal NK. Suddenonset ptosis caused by midbrain neurocysticercosis in 2 children. J Child Neurol 2008;23:334-7.

10. Del Brutto OH, Rajshekhar V, White AC Jr, et al. Proposed diagnostic criteria for neurocysticercosis. Neurology 2001;57:177-83.
11. Prasad R, Thakur N, Mohanty C, Singh MK, Mishra OP, Singh UK, et al. Cysticercal encephalitis with cortical blindness. BMJ case reports 2010;21:1837.

12. García HH, Evans CA, Nash TE, Takayanagui OM, White AC, Jr, Botero D, et al. Current consensus guidelines for treatment of neurocysticercosis. Clin Microbiol Rev 2002; 5:747-56.

13. Singhi P, Dayal D, Khandelwal N. One week versus four week of albendazole therapy for neurocysticercosis in children: a randomized placebo controlled double blind trial. Pediatr Infect Dis J 2003; 22: 268-72.

14. Sinha S, Sharma BS. Neurocysticercosis: a review of current status and management. J Clin Neurosci 2009; 16: 867-76.

15. Baird RA, Wiebe S, Zunt JR, Halperin JJ, Gronseth G, Roos KL. Evidence-based guideline: Treatment of parenchymal neurocysticercosis: Report of the guideline development subcommittee of the american academy of neurology. Neurol 2013; 80: 1424-29. 